\title{
How to Organize a Photocatalysis Conference Online (on a Budget)
}

Mathias Micheel, ${ }^{\mathrm{a}, *}$ Julian Hniopek, ${ }^{\mathrm{a}, \mathrm{b}}$ Elisabeth Hofmeister, ${ }^{\mathrm{a}}$ Carolin Müller, ${ }^{\mathrm{a}, \mathrm{b}}$ Miftahussurur Hamidi Putra, ${ }^{\mathrm{c}}$ Ludwig Schwiedrzik, ${ }^{\mathrm{d}}$ and Pascal Wintergerst ${ }^{\mathrm{e}}$

Originally planned as an on-site meeting, the inaugural CataLight Young Scientist Symposium (CYSS) took place as a fully online conference in November 2020. Dedicated to various aspects of photocatalysis, namely synthesis, theory, characterization, and application, CYSS aimed to provide a stage for early-career scientists to connect to each other and present their research to peers in the field. While still keeping a traditional on-site conference format including both plenary and poster sessions, several minor and major changes had to be applied to the format to deliver a full experience. In this report, we highlight key steps in the organization of such an online conference, laying a focus on using mostly open source software to minimize costs, and discuss differences to both on-site and other online conference formats.

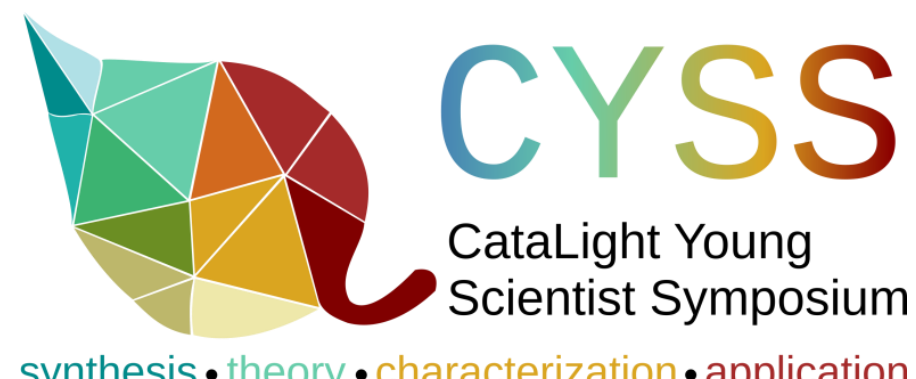

synthesis • theory $\bullet$ characterization $\bullet$ application

\section{Introduction}

The CataLight Young Scientist Symposium (CYSS, www.cyss2020.de) was planned as an on-site conference in Ulm, Germany, in autumn 2020. Its board of organization consists of PhD students and early-career, i.e. within the first two years after obtaining their $\mathrm{PhD}$, postdocs who perform research within the framework of the Deutsche Forschungsgemeinschaft funded Transregional Collaborative Research Center TRR234, CataLight [1], a project with particular emphasis on artificial photocatalysis and the photoactivated hydrogen evolution reaction. Similarly, the target audience of CYSS were young scientists at an early career stage, i.e., PhD students, postdocs, and graduate students, working in the fields of photocatalysis and photochemistry.

However, when faced with the ongoing Covid-19 crisis in Europe and Germany in the early planning stage of the conference, we decided to move the format from completely on-site to fully online. This move was not unprecedented, as several entirely online conferences already existed [2], established on-site conferences switched to an online format [3-5], or novel online formats emerged [6,7]. Advantages of online conferences are numerous and have recently been summarized by Roos et al. [2], and include low costs, as no venue or catering has to be organized, environmental friendliness, with onsite conferences producing considerably more carbon dioxide and waste than online ones due to long-distance travelling [8-11], and time efficacy, as participants may join or opt-out from any talk they would like to listen or not listen to at a whim and can concentrate, e.g., on child care. The most severe disadvantage of such a format is probably the high level of anonymity, as participants rarely meet "face to face", and the associated lack of networking, which is especially vital in the career planning of young researchers [12].

When faced with the organization of an online conference, we quickly realized the lack of a unified organizational standard on how to organize one. In fact, it seemed as many online 
conferences use a combination of several, often proprietary or closed-source, services to offer a full conference experience: Google Forms to obtain registration data, Zoom to host talks, and Twitter to host a poster session [13]. Additionally, it has been argued that the higher technological complexity of online formats might make them even more expensive than on-site conferences [14], even though some digital formats reported reduction of costs to $50 \%$ of onsite ones [15], calling the economic feasibility of online formats into question.

In this report, we want to share our experiences in the organization of CYSS. Special emphasis was laid on the use of open source software (OSS) solutions, which drastically decrease the cost of organization, and data privacy, which is not guaranteed when using third party apps like GoogleForms or Twitter. Additionally, we discuss both synchronous and asynchronous means of inter-participant communication, which could eliminate the condition of meeting at the same time and space. We also include feedback by conference participants, collected in an postconference poll $(n=30)$, and provide an outlook for the future of scientific (chemistry) conferences and how they can profit from including online meetings.

\section{Conference Setup}

For the conference website, we employed Indico (www.getindico.io), an OSS conference organization software, which proved to be a powerful tool in the conference's organization. Indico provided a registration interface, an abstract submission review platform, allowed for sending out mass emails to all participants, and provided an ordered timetable for the conference. Thus, we consider it a fitting platform for all kinds of online meetings, erasing the need for external services such as Google Forms. In addition, one could create surveys directly on the platform, allowing for centralized and facile acquisition of participants' feedback.

As a digital substitution of coffee breaks, we implemented an instance of Mattermost (https://mattermost.com), an OSS chat service in which people can either communicate directly with each other or group-chat in different "channels". While we used Mattermost for our poster session and post-plenary-session discussions (vide infra), we also additionally provided one central channel for socializing and one for technical support. We envisioned that people could have "digital coffee breaks" there and discuss on- and off-topic matters.

For the talks, we employed Zoom, as it is among the most used platforms during the current Covid-19 crisis and we had a Zoom Pro licence made available from the Friedrich Schiller University Jena. While we also tested an OSS alternative, Jitsi, we encountered insurmountable technical challenges.

In general, the predominant use of OSS for the organization enabled CYSS to stay free from proprietary third-party services, which are either prohibitively costly (often up to $1000 €$ per conference) or are problematic in their use of participant data. To run Indico and Mattermost, a cheap server (60€ per year) was more than enough to accomodate the needs of CYSS.

Using OSS for video conferencing, on the other hand, proved much more difficult. While Zoom has been criticized for its poor data privacy, it is much more costly to run a video conferencing system due to excessive bandwidth requirements for higher participant numbers. Even a generously equipped server with $1 \mathrm{Gbit} / \mathrm{s}$ bandwidth will only accommodate ca. 50 participants with video simultaneously and the requirements scale superlinearly with the number of participants. This makes the use of OSS for large scale video conferencing cost prohibitive and cannot be solved on a single conference level. DFNconf (https://www.conf.dfn.de/), which was used for the digital poster presentation (vide infra), is similarly to Zoom not open and restricted to members of German research institutions.

An additional hurdle in using OSS systems is that they need to be set up and configured by the organizers themselves. While this has become much easier in the last years, due to the availability of virtual servers and very good tutorials for Indico and Mattermost, it still requires a level of familiarity with server infrastructure and web-hosting. This requires at least one member of the organizing committee with experience in these fields. 


\section{Advertising and Participants}

CYSS was advertised by direct invitations to individual researchers working in the field of photocatalysis, emails to institutional and international mailing lists, and via twitter. Twitter is a good option for advertising, as it is one of the main social media platforms for academics, allows for tagging of speakers and institutes to extend the audience of advertising, and also functions as a secondary networking space outside of the CYSS Mattermost hub and after the conference.

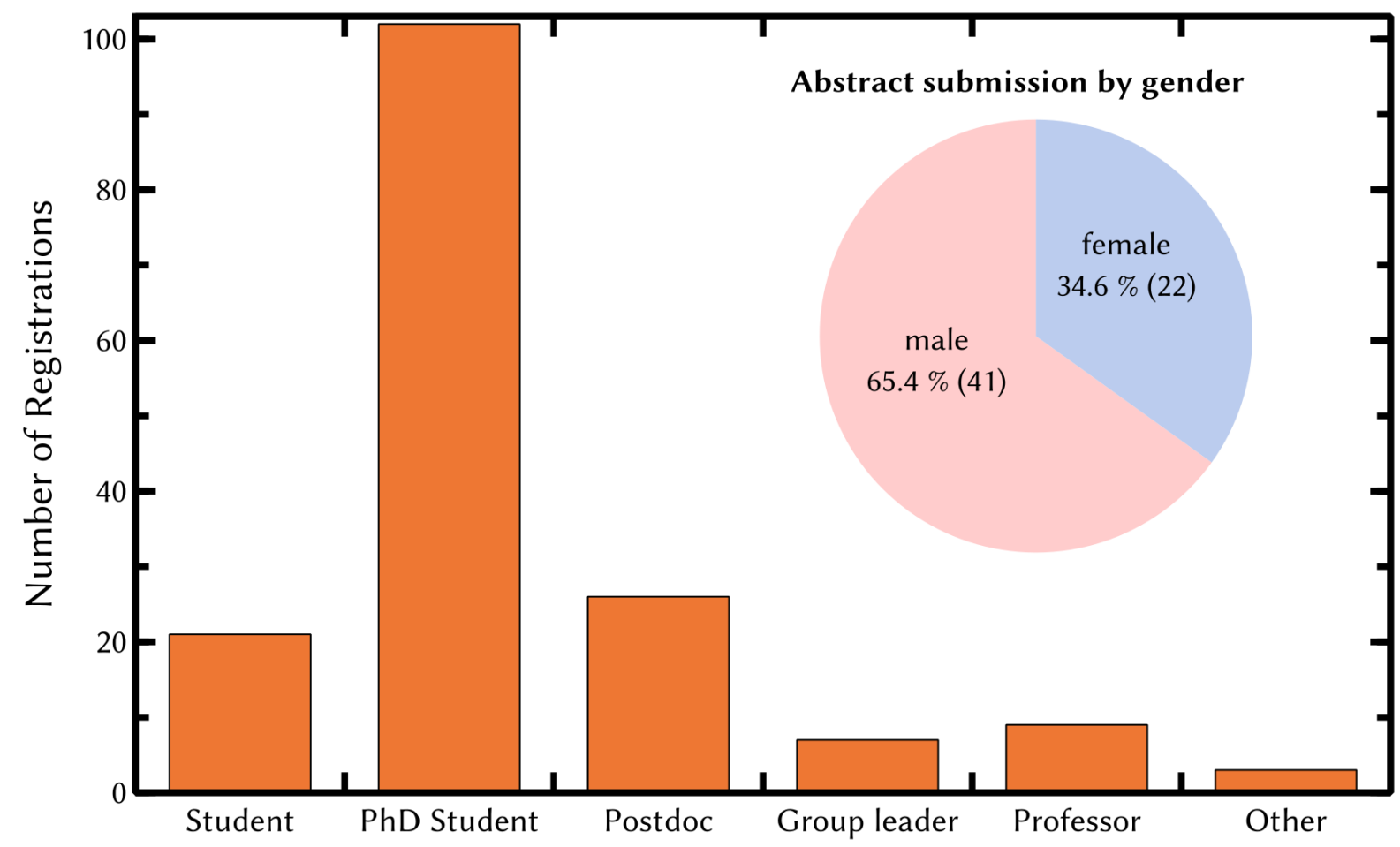

Figure 1. Evaluation of registrants by profession (self-declared by registrants) and number of abstracts submitted by gender (read by the organizers).

In total, nearly 180 people from 31 countries registered for the conference with over 60 submissions for talks and/or poster presentations. The majority of these registrations came from researchers who have yet to obtain their doctoral degree, while professors and group leaders were only present as a minority, with most of them consisting of invited speakers (Figure 1). We estimate that c. 120 people actively participated in the conference, based on the number of unique registrations on our Mattermost chat server, including 7 invited speakers, 29 plenary speakers, and 33 poster presentations. From the 63 submitted abstracts, roughly a third was submitted by women. The male-to-female ratio at CYSS roughly corresponds to the ratio in chemistry in general [16].

\section{Plenary Sessions}

The entire conference took place on six days over the course of three weeks, with sessions starting each Tuesday and Thursday at $3 \mathrm{pm}$ MET. This time allowed participation in particular from Europe, but potentially even from as far as the US West coast (6 am) or Tokyo (11 pm). Each day was dedicated to one specific topic, namely synthesis, characterization, theory, and application. Each session began with a 30 min lecture by an invited speaker, followed by two 20 min talks, a 20 min break, and three more 20 min talks. Each session was chaired by one of the organizers. The focus on small timeslots spread out over several days enabled the incorporation of the conference into a normal workday, without being as interrupting as a full day conference 
would be as well as avoiding "Zoom fatgue" . Participation during each session varied between 45 and 80 people.

After each talk, participants could write their questions in the Zoom integrated chat, which were then read out by the chair. Additionally, we set up the aforementioned, separate Mattermost server where participants could write their questions even after a talk and engage in discussions with the speakers and other participants. We hoped that this chat platform could serve as a replacement to traditional coffee breaks.

We identified several advantages, but also drawbacks in this organization of plenary sessions. The text-based questions, which were centrally read out by the chair, greatly simplified the discussion process, as participants did not need audio equipment themselves to participate. On the other hand, we felt that the lack of "direct" discussions between the presenter and the asking participant created a lack of engagement. Additionally, if the question contained typing errors, it was sometimes hard for the chair to identify the intended meaning, while the questioner was not able to easily clarify his question and/or ask direct follow-up questions. Even though participants were encouraged to type their questions already during the talk, most people opted to wait for the talk to end to write down their questions. Thus, there was sometimes a small waiting period between the ending of the talk and the beginning of the discussion. Last, even though there was a dedicated channel on our Mattermost service, only few people used this opportunity to engage in after-presentation discussions, while at on-site conferences, coffee breaks are often used to engage in scientific discussions. However, we do not know if participants opted to open a private chat with presenters to discuss details of their talk and research presented.

Before each session, the respective session's chair offered all presenters an optional tech-check to see if their audio and video equipment was working without fault. Additionally, we tested the setup and the Zoom chat ourselves intensively in advance, including presentations to run during breaks, moderation of participants, and permissions of participants, e.g. screen-sharing. Accordingly, there were only little technical problems encountered during the conference. Still, the time planned for each talk was scheduled to include small buffers for technical problems or overrunning discussions, which proved to be very helpful on all days of conference.

\section{Poster Sessions}

The poster session was organized using the Mattermost server as well. For each poster, a separate chat channel was created, where people could text chat with the presenter. In addition, DFNconf video chat rooms were provided for each poster. Thus, participants could engage with the presenters "face to face" via video chat or asynchronously using the text chat. We believe this approach has several advantages: First, it does not require registration with an external website that might be geoblocked by the local government, e.g. twitter. Second, text chat is very technologically robust, requiring neither a high-speed internet connection nor webcam or microphone.

We found that text chat facilitates informal discussions between participants on a level comparable to on-site poster sessions, while in comparison, the video chat rooms we provided were used by far fewer participants. Text chat allowed participants to engage with several presenters at the same time by posting questions in chat, then moving on to another poster while awaiting an answer. The separate chat channels created for each poster on our Mattermost server also allowed presenters to look at other posters when not answering questions without fear of missing any discussion of their own work that might be taking place in their absence. Finally, the chat channels were very convenient for us as organizers to set up and moderate, compared to the effort of setting up multiple video chat rooms and making them easily accessible to all participants. One drawback that occurred in regard to the poster presentation on chat was that very few presenters did not appear. This could be because of a big time difference where the poster presentation did not start until after midnight, e.g., for Asian participants.

The Mattermost platform also has an integrated poll feature, and we allowed that all participants of the poster session voted for their three favourite posters per session, awarding the poster prize (sponsored by a scientific book publisher) as a "community prize". Poster 
presenters were asked to prepare their poster in an aspect ratio of 16:9 for best visibility on a computer screen [18].
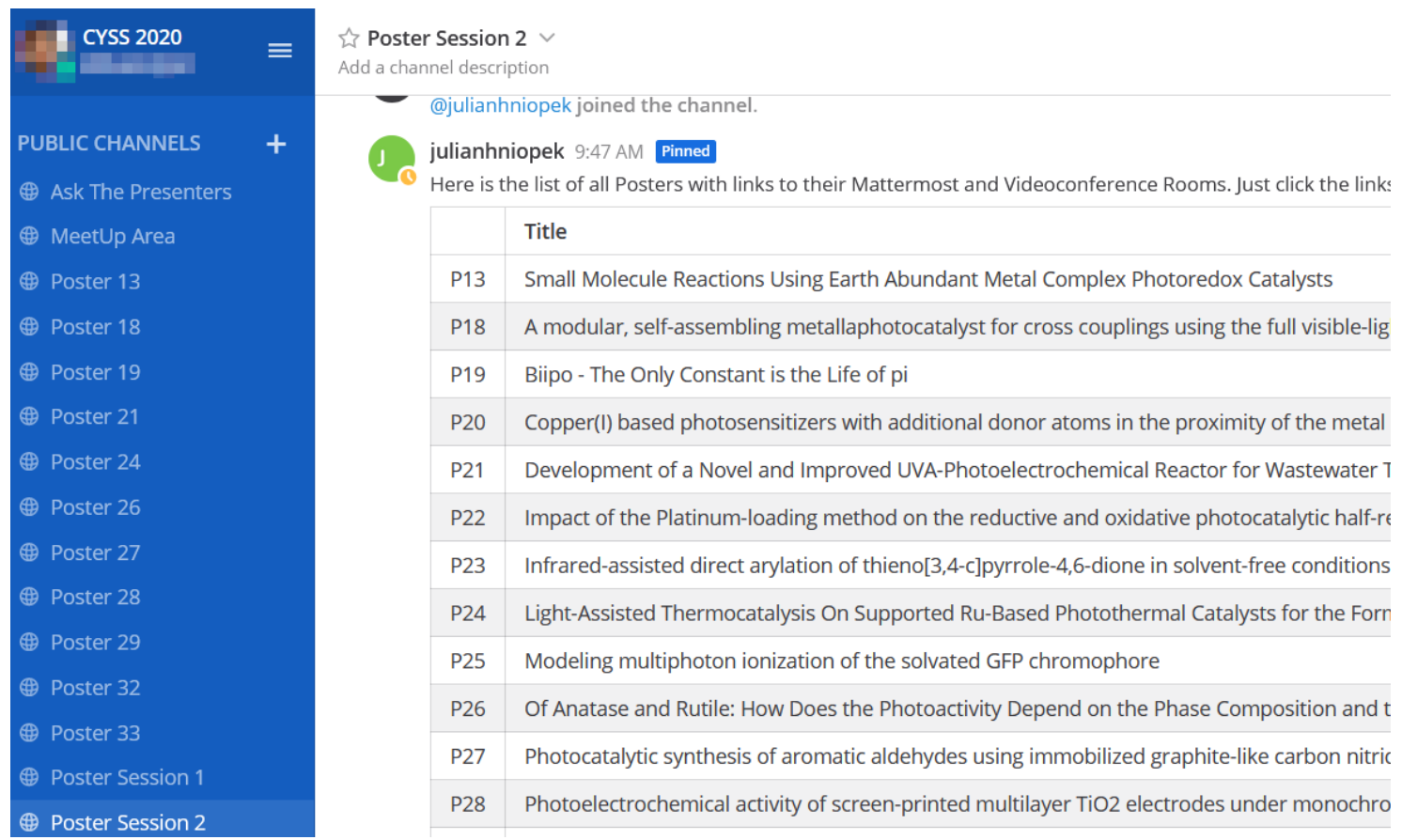

Figure 2. Poster session in Mattermost. In the left bar, participants can switch rooms to see different posters and engage with the presenters. In a central breakout room, a table lists all posters with title, presenter, link to the room within Mattermost and direct link to the DFNconf video chat (cut at the right edge).

\section{Participants' Feedback}

Satisfaction of participants with CYSS 2020 was generally high as was monitored by a postconference survey (Figure 3A). However, some distinct points of improvement can be made out. Even though participants felt that they could connect to people they did not know beforehand, the overall opinion was that networking differed from on-site conferences (Figure 3B). It has to be emphasized that the question did not ask whether it was worse or better. As networking is one of the main aspects of a scientific conference, this needs to be addressed. Similar to on-site conferences, online meetings could also offer social, but digital activities. For example, a virtual "pub quiz" could be organized, where interested participants are organized in randomly selected groups and interact with previously unknown people, and in which questions are asked from the topic(s) covered by the conference. Alternatively, depending on the conference's audience, online computer games could be played jointly in small groups, such as Among Us. While not replacing on-site socializing, such programs could greatly enhance the online conference experience. Additionally, virtual meeting rooms such as Mozilla Hubs (hubs.mozilla.com) present an interesting option for providing networking opportunities at online events. [17] Finally, central Q\&A sessions with the invited speakers could be organized, during which participants can ask both scientific questions as well as for career advice in their respective field.

While a majority of participants would also visit a next iteration of CYSS irrespective of the format it takes place in, a purely online format is the preferred way (Figure 3C). Notably, a purely offline format appears to be the second most-preferred format, while a mixture of both on- and offline seems to be met with reservations. It did not come as a surprise that a purely online meeting would be the preferred choice, as it allows participation to the conference irrespective of travelling costs, which is advantageous for international participants. "Mixed" conferences, on the other hand, are met with more scepticism, probably because such formats have not been implemented widely in the chemistry community as of now 

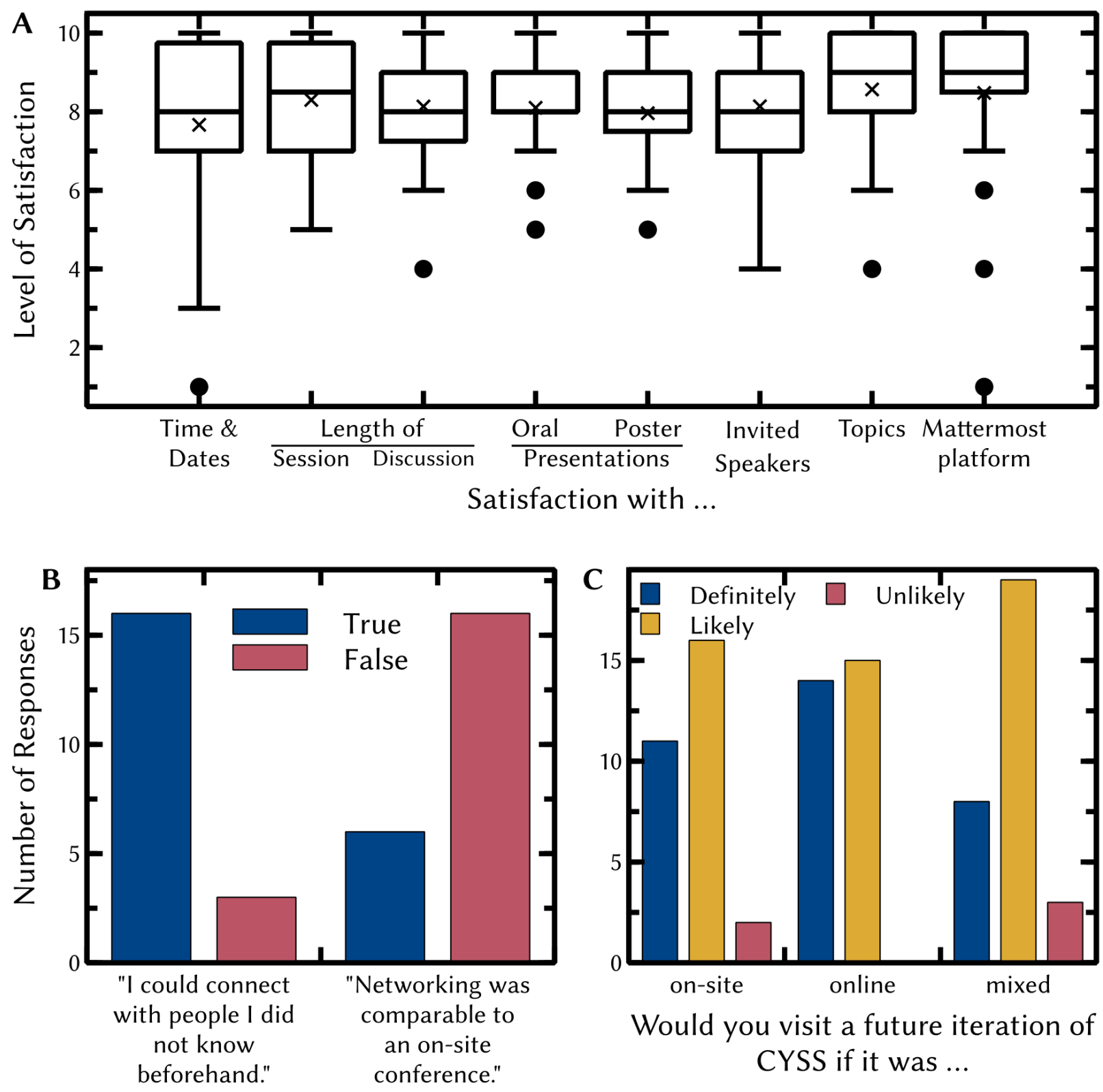

Figure 3. Evaluation of the post-conference survey $(n=30)$. (A) Participants were asked for their level of satisfaction from 1 (lowest) to 10 (highest). For the Mattermost platform, 6 participants responded that they did not use it. Data is presented within a median box plot (IQR: 25th - 75th percentile box, median line, standard deviation whiskers, mean as cross) with only outliers presented as data points. (B) Participants were asked whether they agreed or disagreed with the statements. (C) Participants were asked if they would visit a future iteration of CYSS if it was purely on-site, purely online, or a mixed form (which was not further described).

\section{Conclusion}

While 2020 (and probably a huge part of 2021 still) introduced online conferences to an immensely huge audience, future on-site meetings should keep in mind the lessons learnt from online meetings such as CYSS. Digital socializing hubs such as Mattermost can be implemented irrespective of the format to promote socializing between participants and help in the dissemination of organisational details, while offering the possibility of online participation, such as remote poster sessions, at the same time. Hopefully, funding bodies and research associations will create more safe (with regards to data privacy) and, potentially, OSS digital infrastructures, as to avoid using commercial, closed applications such as Zoom, in the future. 
Thus, online meetings could present not an alternative or substitution, but support and supplementation to already established on-site experiences; we therefore plan to maintain an online element to future iterations of CYSS.

\title{
Author Information
}

\author{
Affiliations \\ ${ }^{a}$ Leibniz-Institute of Photonic Technology (IPHT), Department Functional Interfaces, Albert- \\ Einstein-Straße 9,07745 Jena, Germany \\ Institute of Physical Chemistry, Friedrich Schiller University Jena, Helmholtzweg 4, 07743 \\ Jena, Germany \\ ${ }^{c}$ Institute of Theoretical Chemistry, Ulm University, Albert-Einstein-Allee 11, 89081 Ulm, \\ Germany \\ ${ }^{\mathrm{d}}$ Institute of Theoretical Chemistry, Faculty of Chemistry, University of Vienna, Währinger Str. \\ 17, 1090 Vienna, Austria \\ ${ }^{\mathrm{e}}$ Institute of Inorganic Chemistry I, Ulm University, Albert-Einstein-Allee 11, $89081 \mathrm{Ulm}$, \\ Germany
}

\section{Corresponding Author}

*Mathias Micheel (mathias.micheel@leibniz-ipht.de)

\section{ORCID}

Mathias Micheel: 0000-0002-5017-3511

Julian Hniopek: 0000-0002-8652-8812

Miftahussurur Hamidi Putra: 0000-0001-5363-4152

Ludwig Schwiedrzik: 0000-0002-9361-1032

\section{Funding}

Financial support is acknowledged by the German Research Foundation (DFG)-project number 364549901-TRR234 (CataLight). L.S. acknowledges support from the Fonds zur Förderung der wissenschaftlichen Forschung (FWF), project I3987-N28. We kindly thank Royal Society of Chemistry (RSC) Publishing for providing a poster prize.

\section{Conflicts of interest}

The authors declare no conflicts of interest.

\section{References}

1 CataLight.eu Available online: https://www.catalight.eu/ (accessed on 26 November 2020).

2 Roos, G.; Oláh, J.; Ingle, R.; Kobayashi, R.; Feldt, M. Online Conferences - Towards a New (Virtual) Reality. Computational and Theoretical Chemistry 2020, 1189, 112975, doi:10.1016/j.comptc.2020.112975.

3 Busse, B.; Kleiber, I. Realizing an Online Conference: Organization, Management, Tools, Communication, and Co-Creation. International fournal of Corpus Linguistics 2020, 25, 322-346, doi:10.1075/ijcl.00028.bus.

4 Falk, M.T.; Hagsten, E. When International Academic Conferences Go Virtual. Scientometrics 2020, doi:10.1007/s11192-020-03754-5. 
5 Seery, M.K.; Flaherty, A.A. Ten Tips for Running an Online Conference. F. Chem. Educ. 2020, 97, 2779-2782, doi:10.1021/acs.jchemed.0c00833.

6 Reshef, O.; Aharonovich, I.; Armani, A.M.; Gigan, S.; Grange, R.; Kats, M.A.; Sapienza, R. How to Organize an Online Conference. Nature Reviews Materials 2020, 5, 253-256, doi:10.1038/s41578020-0194-0.

7 Ramilo-Gomes, F.; António, J.P.M.; Mendes, M.I.P.; Gomes, R.F.A. MedChemTrain E-School 2020: Event Highlights and Insights into Virtual Symposium Organization. ChemMedChem 2020, doi:10.1002/cmdc.202000450.

8 Burtscher, L.; Barret, D.; Borkar, A.P.; Grinberg, V.; Jahnke, K.; Kendrew, S.; Maffey, G.; McCaughrean, M.J. The Carbon Footprint of Large Astronomy Meetings. Nature Astronomy 2020, 4, 823-825, doi:10.1038/s41550-020-1207-z.

9 Desiere, S. The Carbon Footprint of Academic Conferences: Evidence from the 14th EAAE Congress in Slovenia. EuroChoices 2016, 15, 56-61, doi:10.1111/1746-692X.12106.

10 Jäckle, S. WE Have to Change! The Carbon Footprint of ECPR General Conferences and Ways to Reduce It. Eur Polit Sci 2019, 18, 630-650, doi:10.1057/s41304-019-00220-6.

11 Klöwer, M.; Hopkins, D.; Allen, M.; Higham, J. An Analysis of Ways to Decarbonize Conference Travel after COVID-19. Nature 2020, 583, 356-359, doi:10.1038/d41586-020-02057-2.

12 Oester, S.; Cigliano, J.A.; Hind-Ozan, E.J.; Parsons, E.C.M. Why Conferences Matter-An Illustration from the International Marine Conservation Congress. Front. Mar. Sci. 2017, 4, doi:10.3389/fmars.2017.00257.

13 Randviir, E.P.; Illingworth, S.M.; Baker, M.J.; Cude, M.; Banks, C.E. Twittering About Research: A Case Study of the World's First Twitter Poster Competition. F1000Res 2016, 4, doi:10.12688/f1000research.6992.3.

14 Kelz, H. Wann Wird's Mal Wieder Richtig Tagung? Nachrichten aus der Chemie 2020, 68, 3-3, doi:10.1002/nadc.20204104871.

15 Viglione, G. How Scientific Conferences Will Survive the Coronavirus Shock. Nature 2020, 582, 166-167, doi:10.1038/d41586-020-01521-3.

16 Widener, A. Chemist Diversity by the Numbers Available online: https://cen.acs.org/careers/diversity/Chemist-diversity-numbers/97/i15 (accessed on 11 January 2021).

17 Le, D.A.; Maclntyre, B.; Outlaw, J. Enhancing the Experience of Virtual Conferences in Social Virtual Environments. In Proceedings of the 2020 IEEE Conference on Virtual Reality and 3D User Interfaces Abstracts and Workshops (VRW); March 2020; pp. 485-494.

18 Wickeren, S. van; Kösters, K.; Zhou, Z.; Ying, B. Tagungen und Corona:Quer- statt Hochformat. Nachrichten aus der Chemie 2021, 69, 32-33, doi:10.1002/nadc.20214104049. 